limitations including low participation rates and high costs. Social networking sites, commonly used for communication by young people, present an unique opportunity for innovative recruitment modalities.

Method This is part of a larger feasibility study assessing use of Facebook to recruit subjects for a novel prospective health study, the Young Female Health Initiative (YFHI). Women aged 16 to 25 years, living in Victoria, Australia were eligible to participate. An advertisement was placed on Facebook between May-and October 2010 and was visible to a random sample of eligible women. Women clicking on the advertisement were redirected to our website (http://www.yfhi.org) and invited to provide their contact details. They were contacted by a researcher and asked to complete a survey at the YFHI study site, or to complete the questionnaire online. The survey contained demographic questions, plus sensitive questions about sexual history, experience and knowledge of Chlamydia trachomatis, human papillomavirus (HPV) and HPV vaccines.

Results 551 women responded to the advertisement (recruitment could be scaled up or down by changing the advertising frequency). We enrolled 426 respondents, of whom 278 completed the survey within the time available (50\% at the study site, $50 \%$ online). Respondents' age and geographical distribution (urban, regional, rural) was representative of the target population; women over 18 years were $37 \%$ more likely to enrol and complete the survey than 16-17-year olds $(p<0.05)$. Despite the sensitive nature of some questions, over $98 \%$ of participants found the survey not at all, or only slightly, embarrassing. Overall, $63 \%$ had heard of HPV: of these, $73 \%$ knew that HPV is sexually acquired and $94 \%$ knew that it causes cancer. $78 \%$ had heard of chlamydia: those who were sexually active were more likely to know of chlamydia than virgins $(p<0.01)$, while $63 \%$ knew it could cause chronic pelvic pain, and $86 \%$ that it could cause infertility. This recruitment method also was costeffective (\$USD 20 per compliant participant).

Conclusions Results demonstrate excellent potential for such information and communication technologies (ICT) to engage young women in health research, including those from regional and rural communities, and support the use of ICT in future population-based studies, including sexual health research.

\section{P1-S4.33 EFFECTIVENESS OF RESPONDENT DRIVEN SAMPLING AMONG UNDOCUMENTED CENTRAL AMERICAN IMMIGRANT WOMEN IN HOUSTON, TEXAS, 2010}

\section{doi:10.1136/sextrans-2011-050108.177}

${ }^{1} \mathrm{~J}$ Montealegre, ${ }^{1} \mathrm{~J}$ Risser, ${ }^{1} \mathrm{~B}$ J Selwyn, ${ }^{2} \mathrm{~K}$ Sabin. ${ }^{1}$ The University of Texas School of Public Health, Houston, USA; ${ }^{2}$ WHO, Hanoi, Viet Nam

Background Respondent driven sampling (RDS) is a social research method that uses participants' social networks (SNs) to access members of hidden populations for which there is no sampling frame. RDS is widely used for HIV behavioural research among sex workers, drug users, and other hidden populations. However, it has had minimum application in migrant populations. We used RDS to conduct an HIV behavioural survey among undocumented Central American immigrant women in Houston, Texas (specifically, Guatemalan, Honduran, and El Salvadoran females, ages $18-50$ years, residing in Houston without a valid US visa or residency papers). Here we describe the effectiveness of RDS to recruit members of this population.

Methods Formative research indicated that social ties are mainly formed by country of origin, age, and number of years living in the USA. Measures of effectiveness were survey duration, participants' adoption of the recruitment system, SN density, homophily, and attainment of equilibrium. SN density is the average number of social ties per participant. Homophily is the likelihood that individuals recruit individuals like themselves; scores range from -1 to 1 , where $1=100 \%$ within-group recruitment and $0=100 \%$ random recruitment. Equilibrium is the stable sample composition that indicates independence from initial non-randomly selected participants.

Results Beginning with three initial participants, we recruited a sample of 230 immigrant women over 16 weeks. Participants adopted the recruitment system with reasonable ease (46\% recruited $\geq 1$ peers) and SNs were dense (mean SN size $=20)$. Homophily $(H)$ was moderate by country of origin (Guatemalans: $\mathrm{H}=0.52$; $\mathrm{El}$ Salvadorans: $\mathrm{H}=0.42$ ) and low by age and number of years in the USA $(\mathrm{H} \leq 0.25)$. Equilibrium was attained for all demographic and sexual behaviour characteristics.

Conclusions This study is the first to evaluate RDS in a migrant population. SNs in this population were dense, allowing recruitment to be sustained. While recruitment was moderately influenced by country of origin, women did not affiliate exclusively with those like themselves. This sociometric diversity allowed the sample to attain an equilibrium composition independent of initial participants. Overall, RDS was easy to implement, attained a large sample in a relatively short period of time, and reached an otherwise hidden population. RDS is an effective method for recruiting undocumented Central American immigrant women for HIV behavioural surveys in Houston.

\section{P1-S4.34 CAN RDS BE USED TO RECRUIT UNBIASED SAMPLES FROM THE SAME POPULATION WITH REPEATED SAMPLING?}

doi:10.1136/sextrans-2011-050108.178

J Risser, P Padgett, J Montealegre. School of Public Health, University of Texas, Houston, USA

Background Respondent Driven Sampling (RDS) is a modified snowball sampling method that allows for recruitment of a probability sample, even when we cannot enumerate the population of interest. The National HIV Behavioural Surveillance project, designed to assess trends in risky behaviours, used RDS to recruit injection drug users (IDU) and heterosexuals at high risk of HIV infection (HET). In order to examine changes in behaviours over time, the sampling method must recruit homogeneous populations, so that observed differences across cycles can be attributed to changes in behaviour, rather than to errors in recruitment. This analysis was designed to determine if crude and adjusted measures within and between the IDU and HET cycles, are homogeneous on basic demographic characteristics. Our goal to assess if the samples may be considered to be from the same target population.

Methods Data are from the Houston Texas site of NHBS-IDU 1 and 2 (2005, 2009), and HET 1 and 2 (2006, 2010). Adjusted population prevalence estimates are calculated using RDSAT, adjusting for design effect and sampling characteristics. Estimates were compared using the Mantel Haenszel test for heterogeneity.

Results Comparing IDU1 to IDU2, we found similar population estimates ( $p$ for heterogeneity $>0.05$ ) for age $40-49$ years; Black race; had current health insurance; and currently homeless. The populations differed ( $p$ for heterogeneity $<0.05$ ) by the proportion that: graduated from high school; were arrested in the last year; and visited a doctor in the last year. Comparing HET1 and HET2, we found similar population estimates for the proportions that: had health insurance; visited a doctor in the last year; were arrested in the last year; graduated from high school; and were aged 30-49. The populations differed in the percent: Black; currently homeless; and aged $18-29$.

Conclusion Using RDS to assess behaviour changes over time will be difficult if the study samples do not represent a fixed population. We 
looked at variables we thought would be stable over the two cycles and were surprised to find instability. With these differences, we cannot attribute the observed changes in risky behaviour solely to prevention efforts that occurred between the surveillance cycles. However, RDS remains an exceedingly easy and efficient sampling method and with appropriate multivariable analysis techniques can be used with repeated samples over time.

\section{Epidemiology poster session 5: Transmission dynamic}

\section{P1-S5.01 HETEROSEXUAL ANAL SEX, LUBRICATION, HIV, AND HSV-2 INFECTION AMONG WOMEN}

doi:10.1136/sextrans-2011-050108.179

\author{
${ }^{1} \mathrm{~J}$ Brown, ${ }^{1} \mathrm{~K}$ Hess, ${ }^{2} \mathrm{~S}$ Brown, ${ }^{2} \mathrm{C}$ Murphy, ${ }^{2} \mathrm{~A}$ Waldman, ${ }^{2} \mathrm{M}$ Hezareh. ${ }^{1}$ University of \\ California, Los Angeles, USA; ${ }^{2}$ AIDS Research Alliance, Los Angeles, USA
}

Background Anal intercourse (AI) is associated with increased HIV and STI risk. Anal douching and use of AI lubrication may increase susceptibility to infection and may influence results of trials of microbicides for HIV prevention through effects on the rectal environment and on adherence to investigational microbicidal products. We measured the frequency of heterosexual AI, anal practices and lubricant use and their association with HIV and HSV2 infection among women.

Methods Between October 2008 and June 2009, we recruited women age 18 years and older in Los Angeles. At the enrolment visit and at the 12-month visit, participants underwent a self-administered, web-based questionnaire covering demographic factors, sexual Behaviours, and anal practices. HSV-2 was diagnosed by IgG ELISA, and HIV was diagnosed by rapid ELISA with confirmation by Western blot. Here we report data collected during the enrolment visit.

Results We enrolled 141 women (34\% identified as White, 40\% Black, 26\% Hispanic; the median age was 33 years (IOR=25-44)). Overall, 71\% reported ever having AI, and $8 \%$ reported AI as current means of contraception. Overall, 18\% reported AI over the past month, and among these women, the median number of monthly $\mathrm{AI}$ acts was $2(\mathrm{IOR}=1-3), 50 \%$ reported unprotected $\mathrm{AI}, 8 \%$ reported anal douching, and 58\% reported using lubricant during AI. Commercial lubricants (38\%), saliva (23\%), petroleum jelly (15\%), and lotion (12\%) were the most commonly reported lubricants. The prevalence of HIV was $27 \%$ and HSV-2 was $57 \%$. In multivariable analysis, AI was associated with testing positive for $\mathrm{HIV}(\mathrm{aOR}=7.8$ $(95 \% \mathrm{CI}=1.6$ to 37.6$)$ ) and HSV-2 infection $(\mathrm{aOR}=3.3(95 \% \mathrm{CI}=1.2$ to 9.1)) controlling for age, race, and lifetime sexual partners.

Conclusion AI is associated with HSV-2 and HIV infection, and anal douching and use of anal lubricating products not specifically designed for intercourse is common among women. The frequency and health effects of anal practices and lubricants warrant further study.

\section{P1-S5.02 TOWARDS MORE ROBUST ESTIMATES OF THE PER SEX ACT TRANSMISSION PROBABILITY OF CHLAMYDIA TRACHOMATIS}

doi:10.1136/sextrans-2011-050108.180

C Althaus, N Low. University of Bern, Bern, Switzerland

Background Estimating the per sex act transmission probability of Chlamydia trachomatis is necessary to assess an individual's risk of infection and re-infection after sexual contacts with chlamydiapositive partners. Katz (1992, Stat Med) made one of the first attempts to estimate the probability of chlamydial transmission based on data about the proportions of concordant (both partners infected or uninfected) and discordant (only one partner infected) couples tested for chlamydia. This provides a cross-sectional per partnership transmission probability but did not address the duration of sexual partnerships and the spontaneous clearance of chlamydia. Mathematical models can be used to take these complexities into account.

Methods We developed a simple mathematical model of chlamydia transmission that incorporates the formation and dissolution of sexual partnerships. Chlamydia can be transmitted from an infected to an uninfected partner throughout the duration of the partnership. Infected partners can also clear chlamydia spontaneously. The model was fitted to published data from a cross-sectional study of chlamydia infection status in heterosexual couples at a US sexually transmitted diseases clinic. The model was further parameterised with study data about overall chlamydia positivity and the number of sexual partners in the last 6 months.

Results The data showed similar proportions of infected female partners of infected men and infected male partners of infected women, suggesting similar transmission probabilities. Assuming an average infectious duration of 12 months for women and men, we obtained best-fit estimates for the transmission rate ( 0.0064 per day) and average duration of partnerships (6.0 months) in the study. The transmission rate per day corresponds to a per sex act transmission probability of $4.5 \%$, assuming one unprotected episode of coitus per week in a partnership. Our analysis also shows that these estimates depend on the assumed infectious duration of chlamydia.

Conclusions Our approach shows how the dynamics of sexual partnership formation and disease transmission can be taken into account to derive the daily rate of chlamydia transmission within a partnership. The per sex act transmission probability can then be inferred. These refined estimates will help to parameterise future mathematical models of disease transmission and assess the risk of infection and re-infection after sexual contacts with chlamydiapositive partners.

\section{P1-S5.03 PATTERN OF SEXUALLY TRANSMITTED INFECTIONS (STIS) IN HORMONAL CONTRACEPTIVES AND INTRA- UTERINE DEVICES (IUD) USERS ATTENDING FAMILY PLANNING CLINICS IN IBADAN, NIGERIA}

doi:10.1136/sextrans-2011-050108.181

\section{S A Fayemiwo, A A Fatiregun, R A Bakare. University of Ibadan, Ibadan, Nigeria}

The large majority of women who acquire HIV and other sexually transmitted infections (STIs) are in their childbearing years and are current or potential users of contraceptive methods. Using contraceptives has two main benefits; the primary benefit of preventing unplanned pregnancy and the potential secondary benefit of protecting against sexually transmitted infections (STIs). Unfortunately, the contraceptive with the best records for pregnancy prevention offers little if, any protection against STIs. The study aimed at assessing the pattern of sexually transmitted infections among hormonal contraceptives and intra-uterine contraceptive devices users attending family planning clinic, University College Hospital Ibadan.

Methods This is a cross-sectional study in a population of women using Intra-Uterine Contraceptive Devices (IUCD) and hormonal contraceptive methods attending Family Planning clinics at University College Hospital, Ibadan. Detailed medical history, Endocervical and high vaginal swabs were collected from the participants to establish diagnosis after clinical examination and informed consent.

Results There were 200 participants with a mean age of 31.92 years $(\mathrm{SD}=8.33$, range $=16-55)$. The mean age of sexual debut of participants 